\title{
A Novel Variant in the Endothelin-Converting Enzyme-Like 1 (ECEL1) Gene in an Emirati Child
}

\author{
Abdul Rezzak Hamzeh ${ }^{a}$ Pratibha Nair $^{a}$ Madiha Mohamed $^{b}$ Fatima Saif $^{b}$ \\ Nafisa Tawfiq $^{\text {b }}$ Mohamed Khalifa $^{\mathrm{b}}$ Mahmoud Taleb Al-Ali ${ }^{\mathrm{a}}$ Fatma Bastaki $^{\mathrm{b}}$ \\ ${ }^{a}$ Centre for Arab Genomic Studies and b ${ }^{b}$ Department of Pediatrics, Latifa Hospital, Dubai Health Authority, \\ Dubai, United Arab Emirates
}

\section{Significance of the Study}

- This is a case of an Emirati child with developmental delay, multiple congenital abnormalities, and dysmorphic features due to a novel homozygous mutation in the ECEL1 gene.

\section{Keywords}

Distal arthrogryposis type 5D · Novel mutation · Arab ethnicity · Consanguinity

\begin{abstract}
Objective: The aim of this work was to report a case of an Emirati child who presented with developmental delay and multiple congenital abnormalities that are consistent with distal arthrogryposis type 5D. Clinical Presentation and Intervention: The clinical presentation comprised contractures of the shoulders, elbows, and knees in addition to camptodactyly and neck pterygium. The facial dysmorphic features noted include ptosis and microretrognathia. Importantly, left orchidopexy was also observed and corrected surgically. Whole exome sequencing revealed that the patient is homozygous for the novel c.1184+1G > T variant in endothelin-converting enzyme-like 1 (ECEL1). Conclusion: This is a case of a novel homozygous splice site mutation in the
\end{abstract}

\section{KARGER}

E-Mail karger@karger.com www.karger.com/mpp (c) 2017 S. Karger AG, Basel

\section{Karge}

Open access

This is an Open Access article licensed under the terms of the Creative Commons Attribution-NonCommercial 3.0 Unported license (CC BY-NC) (www.karger.com/OA-license), applicable to the online version of the article only. Distribution permitted for non-commercial purposes only.
ECEL1 gene in a child with a phenotype consistent with distal arthrogryposis type 5D. The child was born to consanguineous Emirati parents heterozygous for the novel ECEL1 mutation.

(c) 2017 S. Karger AG, Basel

\section{Introduction}

Arthrogryposis multiplex congenita is used to describe multiple congenital joint contractures that can be caused by a myriad of etiologies [1]. In fact, arthrogryposis is a clinical finding in hundreds of different disorders as opposed to a specific diagnosis. One major subcategory of arthrogryposis multiplex congenita is distal arthrogryposis (DA), in which congenital joint contractures mainly affect distal extremities without primary neurological and/or muscle disease [2]. Certain clinical criteria have been compiled to delineate DA. For the upper extremities 

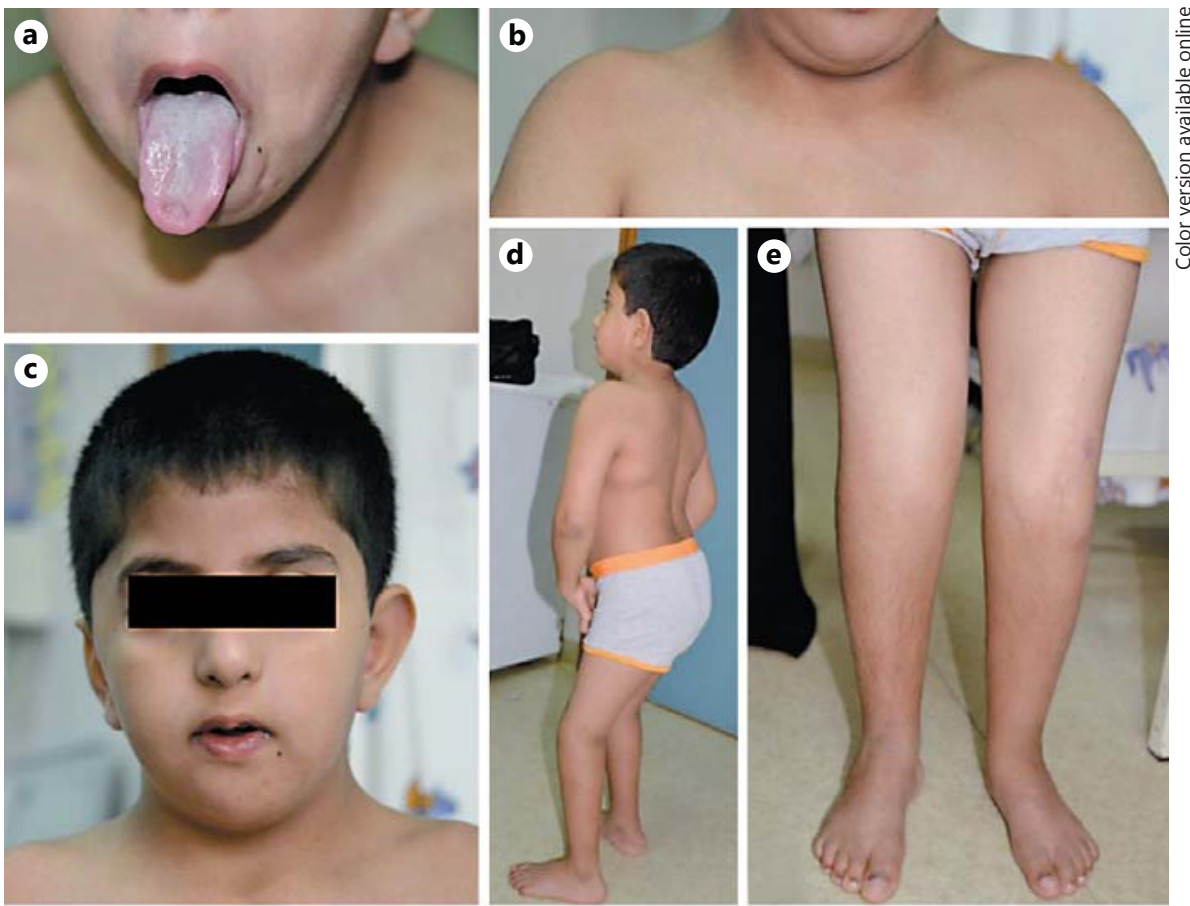

Fig. 1. Clinical features of the patient with homozygous ECEL1 and CHRNG mutations. These features include puckering of the tongue (a) and pterygium between the chin and sternum (b), as well as facial dysmorphic features including plagiocephaly and bitemporal narrowing (c). The patient had mild bilateral shoulder abduction, mild limitation in elbow extension (d), limited knee flexion, left calcaneovalgus (e), and camptodactyly with limited extension of the metacarpophalangeal joints (f). $\mathbf{g}$ An $\mathrm{X}$-ray shows the scoliotic posture with a straightening of the dorsolumbar spine.
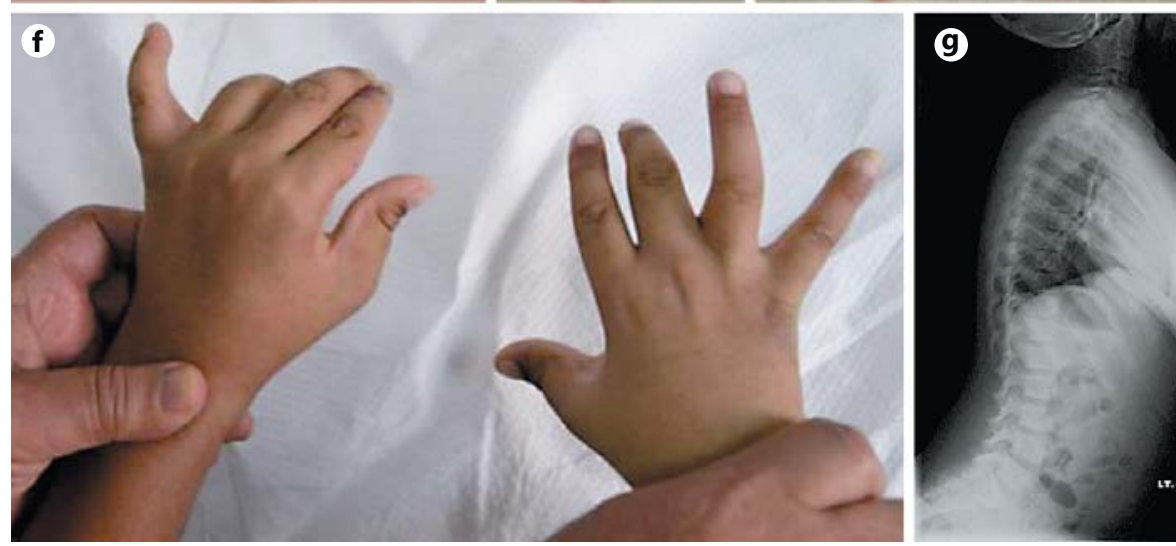

these are camptodactyly, hypoplastic and/or absent flexion creases, overriding fingers, and ulnar deviation of the wrist, and for the lower extremities, talipes equinovarus, calcaneovalgus deformities, and vertical talus. In the absence of family history, individuals must have at least 2 affected body parts in order to be diagnosed with DA. There is considerable genetic as well as clinical heterogeneity among DA cases, and numerous loci have been implicated in the causality of the latter conditions; examples include MYH2, MYH3, MYH8, MYBPC1, TPM2, TPM3, TNNI2, TNNT3, ACTA1, and NEB. Autosomal recessive DA type $5 \mathrm{D}$ (DA5D) is one such condition and it is caused by mutations in genes coding for endothelin-converting enzyme-like 1 (ECEL1).
ECEL1 encodes a member of the M13 family of endopeptidases. These are zinc metalloproteases and type II integral membrane proteins that play a very important role in regulating the activity of neuropeptides and peptide hormones [3]. Studies on ECEL1 in mice have shown that it has a vital role in the terminal branching of motor neurons to the endplate of skeletal muscles. The ECEL1 mutations are linked to DA5D cases and these are characterized by deformities in the upper and lower limbs (camptodactyly, calcaneovalgus feet, and extension contractures of the knee). Certain facial features are linked to this condition, including unilateral ptosis, bulbous nose, and micrognathia [3]. Mere dependence on these clinical features is often misleading because there is a significant 
overlap between the abovementioned symptoms and those of other conditions such as multiple pterygium syndrome (OMIM 265000) caused by mutations in the CHRNG gene [4]. We here report the case of an Emirati child who presented with developmental delay and multiple congenital abnormalities that are consistent with DA5D.

\section{Case Report}

The patient was a 5-year-old male, born to healthy first cousin parents, once removed, who was referred to the genetic clinic for evaluation of developmental delay, dysmorphic features, and multiple congenital abnormalities. No family history of such symptoms exists for the patient. A clinical examination revealed an active and alert boy who walked with extended knees and on the medial side of 1 foot. His height at $119 \mathrm{~cm}$ was between the 25th and 50th centile, while his weight of $27.1 \mathrm{~kg}$ was at the 97 th centile. He was intellectually normal, although his motor skills were slightly delayed.

The facial dysmorphic features included plagiocephaly, bitemporal narrowing, prominent eyes with downslanting palpebral fissures, ptosis, which was more pronounced on the left side, long eyelashes, a broad nasal bridge, microretrognathia, a short, slightly webbed neck with limited head movement, and puckering of the tongue (Fig. 1a-c). Musculoskeletal examination revealed mild bilateral shoulder abduction, mild limitation in elbow extension, limited knee flexion, and left calcaneovalgus (Fig. 1d, e). Camptodactyly was noticed, more on the right than the left, along with limited extension of the metacarpophalangeal joints (Fig. 1f). The thumbs were slightly abducted bilaterally. The calf muscles were atrophic. Radiological examination of the pelvis showed developmental dysplasia of the right hip with abnormal left femoral epiphysis. In addition, a pelvic ultrasound scan revealed an undescended left testis with evidence of left encysted hydrocele of the cord. A scoliotic posture with a straightening of the dorsolumbar spine was seen in the spinal X-ray (Fig. 1g). Mild hypertrophy of the adenoids was noted on X-ray of the postnasal space. Other systems were normal.

The patient underwent surgical correction for the hip dislocation and hyperextension of both knees along with a left orchidopexy. Peripheral blood samples were collected from the proband and his parents. Thereafter, DNA was extracted from blood samples according to standard protocols. Amplicon library construction, exome capture, sequencing, and standard data analysis for the affected child and parents were performed by Sengenics (Kuala Lumpur, Malaysia). The SureSelect Human All Exon V5 exome capture kit was used for library preparation and the samples were sequenced at a minimum of $\times 100$ mean standard coverage using the Illumina HiSeq 2,500 platform. The resulting number of variants was 94,907 . These variants were filtered according to quality, frequency, genomic position, consequences on encoded proteins, pathogenicity, and previously reported associations with the phenotype. Findings from whole exome sequencing were confirmed by Sanger sequencing in the trio. Reference sequence identifiers for wild-type ECEL1 were obtained from GenBank (NCBI reference sequences: NG_034065.1).

DA5D in an Emirati Child with a Novel ECEL1 Mutation

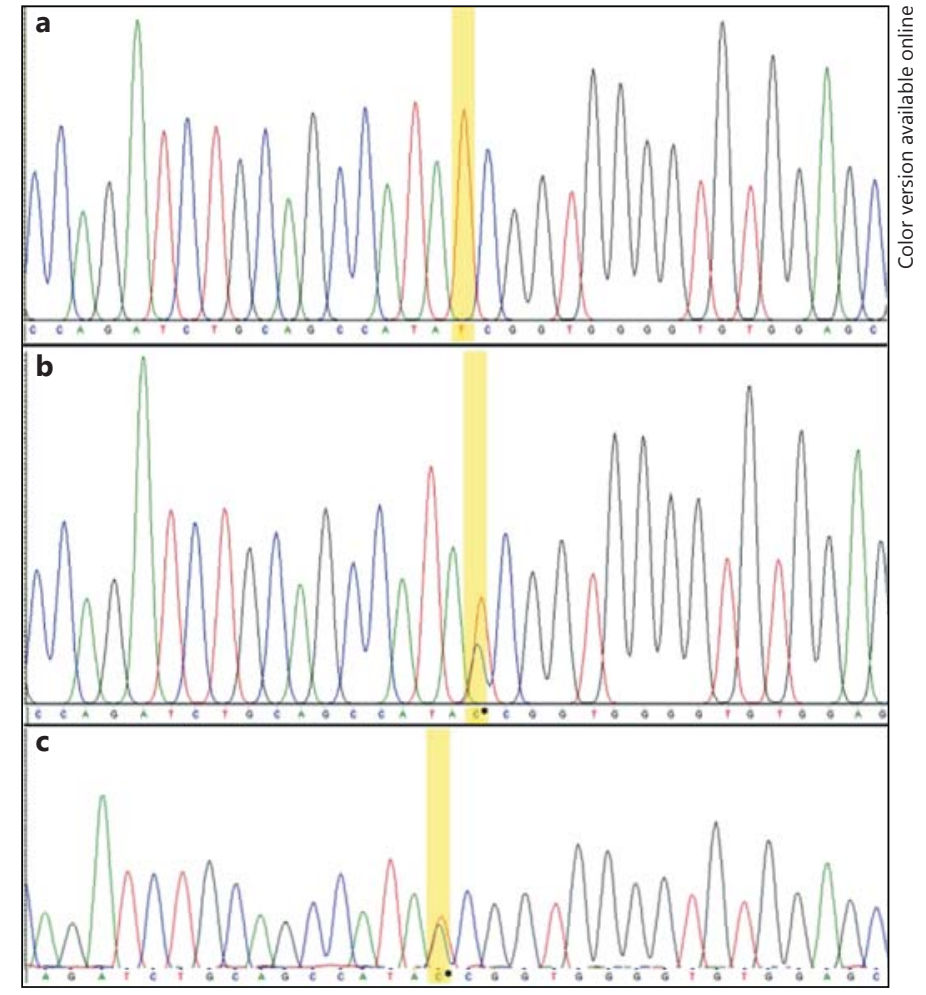

Fig. 2. Sequence chromatograms showing the novel ECEL1 variant in a homozygous state in the patient (a), and in a heterozygous state in each of the parents $(\mathbf{b}, \mathbf{c})$.

The patient harbored a novel single nucleotide variant, c.1184 $+1 G>T$ (GenBank accession No.: KU870924), which occurs at the genomic position chr2:233349181 (assembly GRCh37/hg19) at the beginning of intron 6 in ECEL1 (Fig. 2). This variant was found in a homozygous state in the patient while both parents were heterozygous for it. The variant is predicted to break the WT donor site, which will probably disrupt normal splicing as per prediction algorithms in the Human Splicing Finder and CADD. The variant was not reported in the Exome Aggregation Consortium, Exome Sequencing Project, or in the 1000 Genomes browser. It was also not found in the GalaxC ${ }^{\mathrm{TM}}$ Allele Frequency Database, which contains $>2.5$ million unique Middle Eastern pathogenic mutations and variants.

\section{Discussion}

In this case, the patient harbored a homozygous variant that is located at a splice donor in the ECEL1 gene, hence it might affect proper splicing, as was predicted by $2 \mathrm{R}$ package splicing prediction tools: ADA and Random Forest [5]. The latter findings were corroborated by both Human Splicing Finder and CADD, which yielded a

Med Princ Pract 2017;26:195-198 
PHRED scaled score of 24.8. Numerous mutations in the ECEL1 gene have been reported recently, and, with the spectrum of clinical features of the associated condition, DA5D is becoming more established with each one [6-8]. Many of the characteristic features of DA5D are present in this reported case.

In addition to the ECEL1 variant, the patient was found to harbor a homozygous variant in the $C H R N G$ gene: c. $195+29 \mathrm{C}>\mathrm{T}$. It is unlikely that the latter intronic variant is involved in the overall phenotype of the patient as indicated by the results of various in silico prediction tools. According to the prediction made by CADD, the CHRNG variant had no significant functional consequences, while certain prediction algorithms of Human Splicing Finder suggested that the variant may create an intronic ESE site with potential alteration of splicing (http://www.umd.be/ HSF3/). Even though certain symptoms, such as pterygia and cryptorchidism, are more associated with $C H R N G$ mutations, they are not exclusive to the Escobar type of multiple pterygium syndrome and have occasionally been reported with ECEL1 mutations [9]. The importance of having functional ECEL1 and CHRNG for normal formation of the neuromuscular junction is much clearer than the exact mechanisms underlying the phenotypes caused by their mutations. Shedding light on more cases of such mutations will undoubtedly help elucidate the abovementioned mechanisms, especially if in silico studies are supported by functional ones.

\section{Conclusion}

This is a case of a novel homozygous splice site mutation in the ECEL1 gene in a child with a phenotype consistent with DA5D. The child was born to consanguineous Emirati parents heterozygous for the novel ECEL1 mutation.

\section{Statement of Ethics}

This study was approved by the Institutional Ethics Review Board and conducted with informed written consent from the parents.

\section{Disclosure Statement}

The authors have no conflicts of interest to declare.

\section{References}

1 O'Flaherty P: Arthrogryposis multiplex congenita. Neonatal Netw 2001;20:13-20.

2 Bamshad M, Jorde LB, Carey JC: A revised and extended classification of the distal arthrogryposes. Am J Med Genet 1996;65:277281.

3 McMillin MJ, Below JE, Shively KM, et al: Mutations in ECEL1 cause distal arthrogryposis type 5D. Am J Hum Genet 2013;92:150156.
4 Todd EJ, Yau KS, Ong R, et al: Next generation sequencing in a large cohort of patients presenting with neuromuscular disease before or at birth. Orphanet J Rare Dis 2015;10: 148.

5 Jian X, Boerwinkle E, Liu X: In silico prediction of splice-altering single nucleotide variants in the human genome. Nucleic Acids Res 2014;42:13534-13544.

6 Dieterich K, Quijano-Roy S, Monnier N, et al: The neuronal endopeptidase ECEL1 is associated with a distinct form of recessive distal arthrogryposis. Hum Mol Genet 2013;22:14831492.
7 Patil SJ, Rai GK, Bhat V, et al: Distal arthrogryposis type $5 \mathrm{D}$ with a novel ECEL1 gene mutation. Am J Med Genet A 2014; 164A:2857-2862.

8 Shaaban S, Duzcan F, Yildirim C, et al: Expanding the phenotypic spectrum of ECEL1related congenital contracture syndromes. Clin Genet 2014;85:562-567.

9 Bayram Y, Karaca E, Coban Akdemir Z, et al: Molecular etiology of arthrogryposis in multiple families of mostly Turkish origin. J Clin Invest 2016;126:762-778. 